\title{
Atividade antimicrobiana de novos cimentos endodônticos biocerâmicos
}

\author{
Antimicrobial activity of new bioceramic endodontic sealers \\ Actividad antimicrobiana de nuevos cementos endodónticos biocerámicos
}

\section{Resumo}

Novos cimentos a base de silicato de cálcio prontos para uso (exemplo, Bio-C Sealer e Sealer Plus BC) provaram ser materiais bioativos e biocompatíveis. No entanto, sua atividade antimicrobiana ainda não foi totalmente desvendada. O objetivo deste estudo foi avaliar a atividade antimicrobiana dos cimentos biocerâmicos (Bio-C Sealer, Sealer Plus BC e BioRoot RCS) em comparação com os cimentos à base de resina (AH Plus, Sealer Plus e MTA Fillapex). Os cimentos frescos foram testados contra biofilmes de Enterococcus faecalis, usando testes de contato direto e indireto. As unidades formadoras de colônias foram contadas após 24 horas. Adicionalmente, ensaio cristal violeta foi utilizado para avaliar os efeitos dos extratos dos cimentos na biomassa do biofilme. Medidas de densidade óptica foram usadas para quantificar a biomassa. Os cimentos biocerâmicos apresentaram maior atividade antimicrobiana que os cimentos à base de resina quando em contato direto com biofilmes $(\mathrm{P}<0,05)$. Sealer Plus $\mathrm{BC}$ e Bio-C Sealer apresentaram maior atividade antimicrobiana do que AH Plus no teste indireto $(\mathrm{P}<0,05)$, sugerindo maior difusão desses materiais. $\mathrm{O}$ ensaio cristal violeta revelou que o Sealer Plus BC foi o material mais eficaz na redução da massa do biofilme (P $<0,05)$ apresentando uma diferença significativa com os outros cimentos biocerâmicos. O Bio-C Sealer e o Sealer Plus $\mathrm{BC}$ apresentaram maior atividade antimicrobiana que o AH Plus contra biofilmes de E. faecalis nos testes direto e indireto. Além disso, Sealer Plus BC apresentou o melhor efeito na biomassa do biofilme.

Palavras-chave: Ação antimicrobiana; Endodontia; Materiais obturadores do canal radicular.

\begin{abstract}
New ready-to-use calcium silicate sealers (i.e., Bio-C Sealer and Sealer Plus BC) have proven to be bioactive and biocompatible materials. However, its antimicrobial activity has not yet been fully unraveled. The aim of this study was to evaluate the antimicrobial activity of bioceramic sealers (Bio-C Sealer, Sealer Plus BC and BioRoot RCS) in comparison with resin-based sealers (AH Plus, Sealer Plus and MTA Fillapex). Fresh sealers were tested against Enterococcus faecalis biofilms using direct and indirect contact tests. Colony forming units were counted after 24 hours. Additionally, crystal violet assay was used to evaluate the effects of sealer extracts on biofilm biomass. Optical density measurements were used to quantify biomass. Bioceramic sealers showed greater antimicrobial activity than resin-based sealers when in direct contact with biofilms $(\mathrm{P}<0.05)$. Sealer Plus BC and Bio-C Sealer showed greater antimicrobial activity than AH Plus in the indirect test $(\mathrm{P}<0.05)$, suggesting greater diffusion of these materials. The crystal violet test revealed that Sealer Plus BC was the most effective material in reducing the biofilm mass $(\mathrm{P}<0.05)$ showing a significant difference with other bioceramic sealers. Bio-C Sealer and Sealer Plus BC showed greater
\end{abstract}


antimicrobial activity than AH Plus against E. faecalis biofilms in direct and indirect tests. Furthermore, Sealer Plus $\mathrm{BC}$ showed the best effect on biofilm biomass.

Keywords: Antimicrobial action; Endodontics; Root canal filling materials.

\begin{abstract}
Resumen
Los nuevos cementos de silicato de calcio listos para usar (por ejemplo, Bio-C Sealer y Sealer Plus BC) han demostrado ser materiales bioactivos y biocompatibles. Sin embargo, su actividad antimicrobiana aún no se ha desvelado por completo. El objetivo de este estudio fue evaluar la actividad antimicrobiana de cementos biocerámicos (Bio-C Sealer, Sealer Plus BC y BioRoot RCS) en comparación con cementos a base de resina (AH Plus, Sealer Plus y MTA Fillapex). Los cementos frescos se probaron contra las biopelículas de Enterococcus faecalis utilizando pruebas de contacto directo e indirecto. Las unidades formadoras de colonias se contaron después de 24 horas. Además, se utilizó el ensayo de violeta cristal para evaluar los efectos de los extractos de cemento sobre la biomasa de biopelículas. Se utilizaron medidas de densidad óptica para cuantificar la biomasa. Los cementos biocerámicos mostraron una mayor actividad antimicrobiana que los cementos a base de resina cuando estuvieron en contacto directo con biopelículas $(\mathrm{P}<0.05)$. Sealer Plus BC y Bio-C Sealer mostraron mayor actividad antimicrobiana que AH Plus en la prueba indirecta $(\mathrm{P}<0.05)$, lo que sugiere una mayor difusión de estos materiales. La prueba de violeta cristal reveló que Sealer Plus BC fue el material más eficaz para reducir la masa de biofilm $(\mathrm{P}<0.05)$ mostrando una diferencia significativa con otros cementos biocerámicos. Bio-C Sealer y Sealer Plus BC mostraron una mayor actividad antimicrobiana que AH Plus contra las biopelículas de E. faecalis en pruebas directas e indirectas. Además, Sealer Plus BC mostró el mejor efecto sobre la biomasa de biopelículas.
\end{abstract}

Palabras clave: Acción antimicrobiana; Endodoncia; Materiales de obturación del conducto radicular.

\title{
1. Introdução
}

O emprego de materiais obturadores endodônticos com atividade antimicrobiana é fundamental para auxiliar na eliminação de microrganismos que possam ter permanecido no interior dos canais radiculares após as etapas de instrumentação, seja pela capacidade de matar células bacterianas e/ou romper a biomassa do biofilme (Barros, et al., 2014). Vários tipos de cimentos com diferentes composições estão disponíveis atualmente, incluindo aqueles à base de óxido de zinco e eugenol, resina epóxi, cimentos de resina de metacrilato e materiais à base de silicato de cálcio (Marin-Bauza, et al., 2012; Zordan-Bronzel, et al., 2019). Os cimentos biocerâmicos foram introduzidos no mercado por volta de 2010 com relativo sucesso. Esses cimentos são à base de silicatos e/ou fosfatos de cálcio, os quais chamaram a atenção por suas excelentes propriedades físico-químicas e biológicas (Candeiro, et al., 2016). Esses cimentos apresentam pH alcalino, biocompatibilidade, bioatividade, não toxicidade, estabilidade dimensional, capacidade de selamento, potencial de aumento da resistência radicular após a obturação e eficácia antibacteriana (Candeiro, et al., 2016; Al-Haddad \& Che Ab Aziz, 2016).

Dentre os cimentos à base de silicato de cálcio atualmente disponíveis, tem-se o Bio-C Sealer (Angelus Indústria de Produtos Odontológicos S/A, PR, Brasil). Esse cimento foi lançado recentemente e se apresenta como uma pasta pronta para uso, e tem demonstrado favorecer a expressão de marcadores osteoblásticos e a biomineralização quando em contato com o tecido conjuntivo in vivo (Zordan-Bronzel, et al., 2019; Alves Silva, et al., 2020). Além disso, apresenta atividade antibacteriana (Barbosa, et al., 2020). Na mesma linha de cimentos prontos para uso, está o Sealer Plus BC (MK Dental Life Medical and Products, Porto Alegre, RS, Brasil) que é um material à base de dissilicato e trissilicato de cálcio nanoparticulado. O Sealer Plus BC apresenta biocompatibilidade e potencial bioativo (Benetti, et al., 2019; Alves Silva, et al., 2020), no entanto nenhum estudo demonstrou sua atividade bactericida. Mendes et al. (2018) observaram grande liberação de íons cálcio e pH por esse cimento. O BioRoot RCS (Septodont, Saint Maur-des-Fosses, França) é um cimento biocerâmico de canal radicular à base de silicato tricálcico, apresentado na forma pó/líquida, ou seja, requer mistura manual e possui boas propriedades antibacterianas (Arias-Moliz \& Camilleri, 2016) e biológicas (Siboni, et al., 2017).

A eficácia antimicrobiana dos cimentos biocerâmicos foi avaliada em poucos estudos anteriores, utilizando diferentes métodos microbiológicos e obtendo diferentes resultados (Candeiro, et al., 2016; Bukhari \& Karabucak, 2019; Alsubait, et al., 2019; Bose, et al., 2020; Barbosa, et al., 2020). Estudos recentes revelaram a mesma eficácia dos cimentos biocerâmicos e do 
AH Plus contra biofilmes de Enterococcus faecalis (Candeiro, et al., 2016; Barbosa, et al., 2020). Outros estudos in vitro relataram superioridade dos cimentos biocerâmicos sobre os cimentos à base de resina epóxi (Bukhari \& Karabucak, 2019; Alsubait, et al., 2019; Bose, et al., 2020). Até o momento, estudos avaliando a atividade antimicrobiana de novos cimentos biocerâmicos na forma pronta para uso (Bio-C Sealer e Sealer Plus BC) são escassos (Barbosa, et al., 2020). Apesar das boas propriedades biológicas, fica evidente a importância da avaliação da atividade antimicrobiana dos materiais obturadores endodônticos biocerâmicos frente a biofilmes de microrganismos comumente encontrados no sistema de canais radiculares, principalmente os desenvolvidos mais recentemente. Diante disso, o objetivo deste estudo foi avaliar, in vitro, a atividade antimicrobiana do Bio-C Sealer, Sealer Plus BC e BioRoot RCS (cimentos biocerâmicos) e AH Plus, Sealer Plus e MTA Fillapex (cimentos à base de resina), contra biofilmes de Enterococcus faecalis. Será considerada a hipótese nula de que os cimentos biocerâmicos não apresentarão diferenças entre si e nem em relação aos cimentos resinosos e ao grupo controle.

\section{Metodologia}

\subsection{Cimentos endodônticos}

Foram utilizados os cimentos biocerâmicos Bio-C Sealer, Sealer Plus BC e BioRoot RCS, e os cimentos à base de resina Sealer Plus, MTA Fillapex e AH Plus, este último utilizado como padrão de referência; suas composições estão descritas na Tabela 1. Os cimentos que requerem manipulação foram preparados de acordo com as instruções do fabricante; O Bio-C Sealer e o Sealer Plus BC apresentados na forma pronta para o uso receberam $30 \mu \mathrm{L}$ de água destilada como forma de garantir a umidade necessária para sua presa (Lovato, et al., 2011).

\subsection{Microrganismos}

Utilizou-se uma cepa gram-positiva facultativa de Enterococcus faecalis: ATCC 29212. Os estoques bacterianos foram conservados em meio Brain Heart Infusion (BHI; Kasvi, São José dos Pinhais, PR, Brasil) contendo 20\% de glicerol em freezer a $-80^{\circ} \mathrm{C}$. A cepa bacteriana foi então reativada; $200 \mu \mathrm{L}$ foram inoculados em tubos contendo $10 \mathrm{~mL}$ de caldo BHI em duplicata e incubados a $37^{\circ} \mathrm{C}$ por 18 horas. A pureza da cultura foi verificada por coloração de Gram e o inóculo foi padronizado para uma densidade óptica de 0,1 $(620 \mathrm{~nm})$, para turbidez equivalente a um crescimento de $1-2 \times 10^{8} \mathrm{CFU} / \mathrm{mL}$.

\subsection{Atividade antimicrobiana - testes de contato direto e indireto}

A formação de biofilme foi induzida em membranas de nitrato de celulose de $13 \mathrm{~mm}$ de diâmetro com poros de 0,22 $\mu \mathrm{m}$ (Merck Millipore Ltd, Tullagreen, Cork, Islândia). As membranas foram colocadas na superfície de placas petri contendo ágar BHI. Em seguida, $40 \mu \mathrm{L}$ da suspensão bacteriana foram aplicados nas superfícies das membranas e as placas armazenadas por 48 horas a $37^{\circ} \mathrm{C}$ para formação do biofilme.

Para o teste de contato direto (TCD), 0,15g de cada cimento fresco foi colocado sobre o biofilme formado nas membranas. Para o teste de contato indireto (TCI), uma nova membrana de nitrocelulose estéril foi colocada sobre o biofilme formado antes da inserção do cimento, o qual consiste em um teste restrito por membrana. Para cada cimento, foram utilizadas três membranas, com três repetições em momentos diferentes. O tempo de contato em ambos os testes foi de 30 minutos a $37^{\circ}$ C (Barros, et al., 2014; Viana, et al., 2021). Após a exposição, o cimento foi removido da superfície da membrana no TCD e no TCI a membrana de nitrocelulose com o cimento foi descartada. Os controles positivos foram preparados por meio de membrana com biofilme formado sem nenhum contato com os cimentos. As membranas com biofilme foram transferidas para um microtubo contendo $2 \mathrm{~mL}$ de solução salina estéril e ligeiramente agitadas para remover as células fracamente aderidas. Em seguida, foram transferidos para outro frasco contendo $2 \mathrm{~mL}$ de solução salina e agitados por vórtex durante 1 minuto (30s + 
$15 \mathrm{~s}+15 \mathrm{~s}$ ) intercaladas em banho de gelo entre as agitações. Uma série de diluições em solução salina foi realizada e alíquotas de $10 \mu \mathrm{L}$ de cada diluição foram semeadas em ágar BHI. As unidades formadoras de colônias foram contadas após incubação por 24 horas a $37^{\circ} \mathrm{C}$ e a transformação logarítmica $(\log 10)$ dos dados foi realizada.

Tabela 1. Composição da pasta/pó e pasta/líquido dos cimentos.

\begin{tabular}{|c|c|c|}
\hline & Pasta A / Pó & Pasta B / Liquido \\
\hline $\begin{array}{l}\text { AH Plus } \\
\text { (Dentsply DeTrey GmbH, Konstanz, } \\
\text { Alemanha) }\end{array}$ & $\begin{array}{l}\text { Resina epóxi bisfenol-A } \\
\text { Resina epóxi bisfenol-F } \\
\text { Tungstato de cálcio } \\
\text { Óxido de zircônia } \\
\text { Sílica } \\
\text { Pigmentos de óxido de ferro }\end{array}$ & $\begin{array}{l}\text { Dibenzildiamina } \\
\text { Aminoadamantane } \\
\text { Triciclodecane-diamina } \\
\text { Tungstato de cálcio } \\
\text { Óxido de zircônia } \\
\text { Sílica } \\
\text { Óleo de silicone }\end{array}$ \\
\hline $\begin{array}{l}\text { Sealer Plus } \\
\text { (MK Life, Porto Alegre, RS, Brasil) }\end{array}$ & $\begin{array}{l}\text { Bisfenol A-co-epichlorohydrin } \\
\text { Resina epóxi bisfenol-F } \\
\text { Óxido de zircônia } \\
\text { Silicone e siloxano } \\
\text { Óxido de ferro } \\
\text { Hidróxido de cálcio }\end{array}$ & $\begin{array}{l}\text { Hexametiletilenotetramina } \\
\text { Óxido de zircônia } \\
\text { Silicone e siloxano } \\
\text { Hidróxido de cálcio } \\
\text { Tungstato de cálcio }\end{array}$ \\
\hline $\begin{array}{l}\text { MTA Fillapex } \\
\text { (Angelus, Londrina, PR, Brasil) }\end{array}$ & $\begin{array}{l}\text { Salicilato de resina } \\
\text { Resina natural } \\
\text { Tungstato de cálcio } \\
\text { Sílica nanoparticulada } \\
\text { Pigmentos }\end{array}$ & $\begin{array}{l}\text { Resina diluente } \\
\text { Agregado trióxido mineral } \\
\text { Sílica nanoparticulada } \\
\text { Pigmentos }\end{array}$ \\
\hline $\begin{array}{l}\text { Bio-C Sealer } \\
\text { (Angelus, Londrina, PR, Brasil) }\end{array}$ & $\begin{array}{l}\text { Silicato de cálcio } \\
\text { Aluminato de cálcio } \\
\text { Óxido de cálcio } \\
\text { Óxido de zircônia } \\
\text { Óxido de ferro } \\
\text { Dióxido de silicone } \\
\text { Polietilenoglicol }\end{array}$ & \\
\hline $\begin{array}{l}\text { Sealer Plus BC } \\
\text { (MK Life, Porto Alegre, RS, Brasil) }\end{array}$ & $\begin{array}{l}\text { Óxido de zircônia } \\
\text { Silicato tricálcico } \\
\text { Silicato dicálcico } \\
\text { Hidróxido de cálcio } \\
\text { Propilenoglicol }\end{array}$ & \\
\hline $\begin{array}{l}\text { BioRoot RCS } \\
\text { (Septodont, Saint Maur-des-Fosses, } \\
\text { França) }\end{array}$ & $\begin{array}{l}\text { Silicato tricálcico } \\
\text { Povidona } \\
\text { Óxido de zircônia }\end{array}$ & $\begin{array}{l}\text { Cloreto de cálcio } \\
\text { Policarboxilato } \\
\text { Água }\end{array}$ \\
\hline
\end{tabular}

Fonte: Autores.

\subsection{Atividade antibiofilme: ensaio cristal violeta}

As amostras do teste foram preparadas de acordo com a norma ISO 10993-5: 2009. Após a mistura do cimento, 0,1 g de cada cimento foi imerso em $1 \mathrm{~mL}$ de solução fisiológica e incubado por 24 horas a $37^{\circ} \mathrm{C}$ em câmara umidificada. Os extratos finais foram coletados.

A biomassa do biofilme foi visualizada e quantificada por meio de um ensaio cristal violeta modificado (Barros, et al., 2014). As cepas bacterianas utilizadas foram as mesmas utilizadas no experimento antibiofilme descrito acima. Após agitação por vórtice, alíquotas de $200 \mu \mathrm{L}$ da suspensão bacteriana foram distribuídas em poços de uma placa de microtitulação de 96 poços (poliestireno tratado com cultura de tecidos, fundos planos; Techno Plastic Products, Trasadingen, Suíça) e incubados por 48 horas a $37^{\circ} \mathrm{C}$ para a formação do biofilme. O conteúdo de cada poço foi aspirado e os poços foram lavados 3 vezes com $200 \mu \mathrm{L}$ de solução salina para remover as células fracamente aderidas. Em seguida, foram aplicados $200 \mu \mathrm{L}$ de extratos dos 
cimentos por poço durante 120 minutos e incubados a $37^{\circ} \mathrm{C}$.

Após a remoção dos extratos dos cimentos, cada poço foi lavado 3 vezes com água destilada e $200 \mu \mathrm{L}$ de metanol P.A foram aplicados em cada poço por 10 minutos em temperatura ambiente. Após o período, o metanol foi removido e $200 \mu \mathrm{L}$ de solução de cristal violeta a $0,1 \%$ foram aplicados a cada poço por 10 minutos em temperatura ambiente, para que as bactérias aderentes fossem marcadas. $\mathrm{O}$ excesso de corante foi removido lavando abundantemente com água destilada. As placas foram viradas e secas ao ar, e o corante ligado às células aderentes foi solubilizado com $200 \mu \mathrm{L}$ de ácido acético 33\% durante 10 minutos. Após esse período, a solução de cada poço foi transferida para uma nova placa e a quantificação da biomassa do biofilme remanescente após o tratamento foi lida em leitor de ELISA com absorbância de $590 \mathrm{~nm}$. Para o controle positivo, foi utilizado soro fisiológico em vez da substância de teste. Para o controle negativo, foi utilizado caldo de cultura estéril. Todos os ensaios foram realizados em triplicata em três ocasiões distintas.

\subsection{Análise estatística}

Os dados foram tabulados e submetidos ao teste de Shapiro-Wilks para verificar sua normalidade. A análise unilateral de variância ANOVA e os testes de comparação de Tukey foram usados para testes de contato direto e indireto; e os testes de comparação de Kruskal-Wallis e Dunn foram usados para o teste de atividade antibiofilme cristal violeta. A significância foi estabelecida em $5,0 \%$.

\section{Resultados}

A Tabela 2 apresenta os dados relativos à atividade antimicrobiana dos cimentos testados contra os biofilmes de $E$. faecalis no TCD e TCI. No teste de contato direto, os resultados mostraram que todos os cimentos apresentaram diferença significativa em relação ao grupo controle $(\mathrm{P}<0,05)$; quando comparados ao AH Plus, um parâmetro clínico, todos os outros cimentos apresentaram diferença significativa $(\mathrm{P}<0,05)$. Os cimentos biocerâmicos não apresentaram diferenças entre si, mas apresentaram diferença significativa com os cimentos à base de resina $(\mathrm{P}<0,05)$. Em relação ao TCI, todos os cimentos testados apresentaram diferenças significativas em relação ao grupo controle. Nesta condição o Sealer Plus BC e Bio-C Sealer ofereceram ação maior do que a apresentada pelo AH Plus contra o biofilme de E. faecalis $(\mathrm{P}<0,05)$.

$\mathrm{O}$ ensaio de cristal violeta fornece informações sobre os efeitos dos materiais na biomassa do biofilme. No presente experimento, no extrato de 24 horas (Figura 1), apenas o Sealer Plus BC apresentou leituras de densidade óptica significativamente menores que o controle $(\mathrm{P}<0,05)$. Além disso, Sealer Plus e Sealer Plus BC apresentaram diferença significativa em relação aos biocerâmicos Bio-C Sealer e BioRoot RCS. 
Tabela 2. Média (DP) da contagem bacteriana $\left(\log { }^{10}\right)$ após os testes de contato contra biofilmes de E. faecalis.

\begin{tabular}{cccc}
\hline Cimento & Contato Direto & & Contato Indireto \\
\cline { 2 - 3 } AH Plus & Média (Desvio padrão) & Média (Desvio padrão) \\
Sealer Plus & $10.96^{\mathrm{c}}( \pm 0.27)$ & $9.78^{\mathrm{b}}( \pm 0.58)$ \\
MTA Fillapex & $9.18^{\mathrm{b}}( \pm 0.15)$ & $9.15^{\mathrm{ab}}( \pm 0.14)$ \\
Bio-C Sealer & $9.20^{\mathrm{b}}( \pm 0.07)$ & $9.28^{\mathrm{ab}}( \pm 0.05)$ \\
Sealer Plus BC & $7.96^{\mathrm{a}}( \pm 0.44)$ & $8.16^{\mathrm{a}}( \pm 0.01)$ \\
BioRoot RCS & $8.12^{\mathrm{a}}( \pm 0.05)$ & $8.14^{\mathrm{a}}( \pm 0.08)$ \\
Controle & $7.98^{\mathrm{a}}( \pm 0.20)$ & $9.25^{\mathrm{ab}}( \pm 1.05)$ \\
\hline
\end{tabular}

a,b Diferentes letras sobrepostas indicam diferenças significativas de acordo com a análise unilateral de variância ANOVA e os testes de comparação de Tukey $(\mathrm{P}<0,05)$. Fonte: Autores.

Figura 1. Efeitos dos cimentos obturadores endodônticos em biofilmes de E. faecalis (ATCC 29212). Ensaio de antibiofilme de cristal violeta em microplaca.

\section{HOURS}

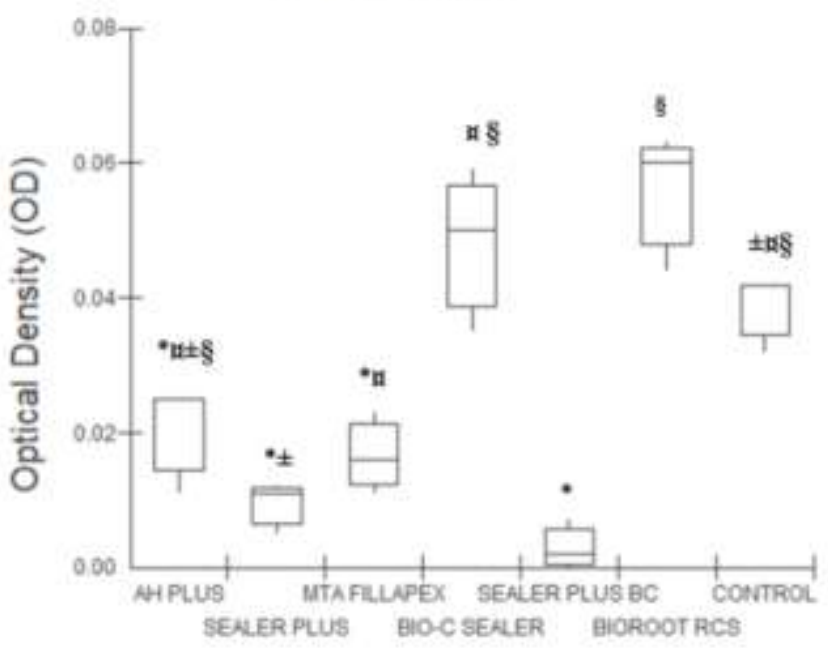

Fonte: Autores.

\section{Discussão}

O presente estudo avaliou a atividade antibiofilme dos cimentos biocerâmicos (Bio-C Sealer, Sealer Plus BC e BioRoot RCS) em comparação aos cimentos à base de resina (AH Plus, MTA Fillapex e Sealer Plus). A hipótese nula foi rejeitada, pois alguns cimentos biocerâmicos apresentaram atividade antimicrobiana diferentes entre si e superiores ao $\mathrm{AH}$ Plus. Os cimentos biocerâmicos vêm chamando a atenção da comunidade científica devido às suas atividades biológicas. Novos cimentos à base de silicato de cálcio prontos para uso foram recentemente introduzidos no mercado, como Bio-C Sealer e Sealer Plus BC. Como diferencial, este foi o primeiro estudo a avaliar a atividade antibacteriana do Sealer Plus BC e a 
comparar cimentos biocerâmicos prontos para uso com um manipulado (ou seja, Bio Root RCS). As limitações deste estudo in vitro incluem o uso de biofilmes de E. faecalis, que são provavelmente mais suscetíveis do que biofilmes multiespécies. Apesar do conhecimento do perfil polimicrobiano das infecções endodônticas, a maioria dos estudos in vitro utilizou biofilmes monoespécies para testar materiais endodônticos, o que possibilitou a comparação de nossos dados com estudos anteriores (Zancan, et al., 2018; Collares, et al., 2018; Swimberghe, et al., 2019).

Os nossos resultados mostraram que o Sealer Plus BC apresentou atividade antimicrobiana e confirmaram a atividade dos cimentos Bio-C Sealer e Bio Root RCS. Nesse esforço, o presente estudo empregou membranas de nitrocelulose por apresentarem fácil padronização e recuperação das células remanescentes dos biofilmes experimentais (Barros, et al., 2014; Kapralos, et al., 2018; Viana, et al., 2021). Além do teste de contato direto, as membranas foram utilizadas para simular a inacessibilidade dos cimentos aos microrganismos, testando a capacidade de difusão de seus componentes contra uma barreira física no teste de contato indireto (Barros, et al., 2014; Kapralos, et al., 2018; Viana, et al., 2021). Quando em contato direto, os cimentos biocerâmicos (Bio-C Sealer, Sealer Plus BC e BioRoot RCS) apresentaram melhor atividade $(\mathrm{P}<0,05)$ contra biofilmes de E. faecalis do que os cimentos à base de resina (AH Plus, Sealer Plus, MTA Fillapex); estudos anteriores confirmam esses achados (Bukhari, et al., 2019; Alsubait, et al., 2019; Bose, et al., 2020). Bio-C Sealer exibiu potencial ação antimicrobiana quando comparado ao AH Plus. A literatura mostra que além da capacidade de induzir a biomineralização após implantação no tecido conjuntivo (Benetti, et al., 2019; Alves Silva, et al., 2020), o Bio-C Sealer tem capacidade de alcalinização atingindo pH de 10 até 21 dias (Mendes, et al., 2018; Zordan-Bronzel, et al, 2019). Sabe-se que pH superior a 9 pode inativar enzimas da membrana celular dos microrganismos, causando perda da atividade biológica ou integridade da membrana plasmática (Barbosa, et al., 2020). Considerando a alta solubilidade do Bio-C Sealer fresco, sua ação antibacteriana pode estar relacionada à liberação significativa de íons cálcio e hidroxila (Zordan-Bronzel, et al, 2019), capazes de inativar a endotoxina bacteriana (LPS). Embora não existam estudos anteriores com Sealer Plus BC; sugere-se que sua ação antibacteriana ocorra devido à sua alta alcalinidade devido à presença de hidróxido de cálcio em sua composição (Mendes, et al., 2018). Segundo Parirock \& Torabinejad (2010), a presença de cálcio pode favorecer um pH alcalino, o que leva a um efeito bioquímico que acelera o processo de cicatrização. No entanto, não se pode excluir a existência de qualquer mecanismo adicional para aumentar a sua atividade. A capacidade antimicrobiana por contato direto do BioRoot RCS pode ser explicada por sua capacidade de sustentar longos períodos de maiores níveis de alcalinidade, o que está de acordo com estudos anteriores (Arias-Moliz \& Camilleri, 2016; Siboni, et al., 2017).

Nessa condição de contato, o cimento resinoso AH Plus apresentou menor atividade antimicrobiana durante o período avaliado, fato também encontrado em estudos anteriores (Poggio, et al., 2017). O AH Plus apresenta baixos valores de pH e solubilidade (Mendes, et al., 2018; Tanomaru-Filho et al., 2019) quando comparado aos cimentos biocerâmicos, o que pode justificar sua menor ação antimicrobiana neste estudo. Os cimentos MTA Fillapex e Sealer Plus também apresentaram ação antimicrobiana significativamente maior do que o AH Plus e o controle. A atividade antimicrobiana do MTA Fillapex é corroborada por outros autores (Shakya, et al., 2016). Estudos mostram que o MTA Fillapex possui alta solubilidade (Tanomaru-Filho, et al., 2019), resultando na liberação de íons $\mathrm{Ca}^{2+}$ e aumentando sua capacidade antimicrobiana. Um estudo anterior também demonstrou que o Sealer Plus apresentou atividade antibacteriana contra E. faecalis e apresentou pH neutro durante todos os períodos testados (Alves Silva, et al., 2020). Os cimentos à base de resina epóxi (AH Plus e Sealer Plus) apresentam menor solubilidade (Tanomaru-Filho, et al., 2019), o que pode estar relacionado ao baixo potencial de alcalinização. Apesar de não ter alta solubilidade, o Sealer Plus possui uma carga considerável de hidróxido de cálcio, que pode ser responsável pela atividade antimicrobiana deste cimento. Valores elevados de $\mathrm{pH}$ assumem particular importância para os cimentos endodônticos, pois a liberação de íons de cálcio estimula a deposição de tecido duro e suas propriedades antibacterianas (Al-Haddad \& Che Ab Aziz, 2016). 
Em relação ao contato indireto, os resultados mostraram uma vantagem para os cimentos biocerâmicos prontos para o uso. Sabe-se que a existência de uma barreira com capacidade de limitar o contato entre os materiais e o biofilme sugere a necessidade de agentes solúveis com capacidade de se espalharem pela umidade do próprio material e do biofilme. Essa característica pode então enfatizar a influência de uma maior solubilidade ou simplesmente capacidade de difusão, devido a sua composição à base de silicatos dependentes de umidade ou um maior tempo de presa, ocorrências que isoladas ou em conjunto podem proporcionar maior alcalinidade em curto prazo, justificando maior difusão de íons hidroxila através da membrana.

A outra abordagem experimental utilizada foi o ensaio de cristal violeta, amplamente utilizado para investigar os efeitos de substâncias na biomassa do biofilme (Alves, et al., 2013; Barros, et al., 2014). Através do uso deste ensaio colorimétrico, altos valores de DO não significam menos capacidade bactericida nas células do biofilme, mas permite avaliar os efeitos na massa do biofilme (Barros, et al., 2014). Este método semiquantitativo cora tanto as células viáveis quanto as mortas, bem como a matriz do biofilme. No presente estudo, a obtenção de extratos de cimento simulou o comportamento do cimento fresco. No extrato de 24 horas, o Sealer Plus BC obteve o menor valor de DO ( $\mathrm{P}<0,05)$, apresentando diferença significativa com o grupo controle. Além disso, Sealer Plus e Sealer Plus BC apresentaram diferença significativa em relação aos cimentos biocerâmicos (Bio-C Sealer e BioRoot RCS), fato esse que pode estar relacionado a presença de hidróxido de cálcio (HC) em suas composições, pois o HC danifica os fosfolipídios da membrana citoplasmática bacteriana promovendo sua oxidação degradativa (Estrela, et al., 1998).

Para o clínico é interessante notar quais materiais, além de outras propriedades, apresentam alta atividade antimicrobiana, principalmente contra biofilmes, podendo colaborar com a limpeza do SCR e com a manutenção da descontaminação alcançada durante o preparo químico-mecânico. Diante dessa observação, os cimentos biocerâmicos, principalmente aqueles apresentados na forma pronta para uso, como Sealer Plus BC e Bio-C Sealer, parecem ter vantagens sobre os outros cimentos testados, aumentando ainda mais o hall de propriedades que contribuem para sua indicação para uso clínico em preenchimento de SCR.

\section{Considerações Finais}

Os cimentos prontos para uso Bio-C Sealer e o Sealer Plus BC apresentaram maior atividade antimicrobiana que o AH Plus contra biofilmes de E. faecalis nos testes de contato direto e indireto. Além disso, o Sealer Plus BC apresentou o melhor efeito na biomassa do biofilme. Considerando as limitações do presente estudo, pode-se concluir que os cimentos endodônticos biocerâmicos apresentaram melhor ação antibacteriana contra biofilmes de E. faecalis do que os cimentos resinosos.

\section{Referências}

Alves, F. R., Neves, M. A., Silva, M. G., Rôças, I. N., \& Siqueira, J. F., Jr (2013). Antibiofilm and antibacterial activities of farnesol and xylitol as potential endodontic irrigants. Brazilian Dental Journal, 24(3), 224-229. https://doi.org/10.1590/0103-6440201302187.

Alves Silva, E. C., Tanomaru-Filho, M., da Silva, G. F., Delfino, M. M., Cerri, P. S., \& Guerreiro-Tanomaru, J. M. (2020). Biocompatibility and bioactive potential of new calcium silicate-based endodontic sealers: Bio-C Sealer and Sealer Plus BC. Journal of Endodontics, 46(10), 1470-1477. https://doi.org/10.1016/j.joen.2020.07.011.

Alsubait, S., Albader, S., Alajlan, N., Alkhunaini, N., Niazy, A., \& Almahdy, A. (2019). Comparison of the antibacterial activity of calcium silicate- and epoxy resin-based endodontic sealers against Enterococcus faecalis biofilms: a confocal laser-scanning microscopy analysis. Odontology, 107(4), 513-520. https://doi.org/10.1007/s10266-019-00425-7.

Al-Haddad, A., \& Che Ab Aziz, Z. A. (2016). Bioceramic-based root canal sealers: a review. International Journal of Biomaterials, 2016, 9753210. https://doi.org/10.1155/2016/9753210. 
Arias-Moliz, M. T., \& Camilleri, J. (2016). The effect of the final irrigant on the antimicrobial activity of root canal sealers. Journal of Dentistry, 52, 30-36. https://doi.org/10.1016/j.jdent.2016.06.008.

Barbosa, V. M., Pitondo-Silva, A., Oliveira-Silva, M., Martorano, A. S., Rizzi-Maia, C. C., Silva-Sousa, Y., Castro-Raucci, L., \& Raucci Neto, W. (2020), Antibacterial activity of a new ready-to-use calcium silicate-based sealer. Brazilian Dental Journal, 31(6), 611-616. https://doi.org/10.1590/01036440202003870

Barros, J., Silva, M. G., Rôças, I. N., Gonçalves, L. S., Alves, F. F., Lopes, M. A., Pina-Vaz, I., \& Siqueira, J. F., Jr (2014). Antibiofilm effects of endodontic sealers containing quaternary ammonium polyethylenimine nanoparticles. Journal of Endodontics, 40(8), 1167-1171. https://doi.org/10.1016/j.joen.2013.12.021.

Benetti, F., Queiroz, Í, Cosme-Silva, L., Conti, L. C., Oliveira, S., \& Cintra, L. (2019). Cytotoxicity, biocompatibility and biomineralization of a new readyfor-use bioceramic repair material. Brazilian Dental Journal, 30(4), 325-332. https://doi.org/10.1590/0103-6440201902457.

Bose, R., Ioannidis, K., Foschi, F., Bakhsh, A., Kelly, R. D., Deb, S., Mannocci, F., \& Niazi, S. A. (2020). Antimicrobial effectiveness of calcium silic ate sealers against a nutrient-stressed multispecies biofilm. Journal of Clinical Medicine, 9(9), 2722. https://doi.org/10.3390/jcm9092722.

Bukhari, S., \& Karabucak, B. (2019). The antimicrobial effect of bioceramic sealer on an 8-week matured Enterococcus faecalis biofilm attached to root canal dentinal surface. Journal of Endodontics, 45(8), 1047-1052. https://doi.org/10.1016/j.joen.2019.04.004.

Candeiro, G., Moura-Netto, C., D'Almeida-Couto, R. S., Azambuja-Júnior, N., Marques, M. M., Cai, S., \& Gavini, G. (2016). Cytotoxicity, genotoxicity and antibacterial effectiveness of a bioceramic endodontic sealer. International Endodontic Journal, 49(9), 858-864. https://doi.org/10.1111/iej.12523.

Collares, F. M., Leitune, V., Portella, F. F., Santos, P. D., Balbinot, G. S., Dos Santos, L. A., Parolo, C., \& Samuel, S. (2018). Methacrylate-based root canal sealer containing chlorexidine and $\alpha$-tricalcium phosphate. Journal of biomedical materials research. Part B, Applied biomaterials, 106(4), 1439-1443. https://doi.org/10.1002/jbm.b.33946.

Estrela, C., Pimenta, F. C., Ito, I. Y., \& Bammann, L. L. (1998). In vitro determination of direct antimicrobial effect of calcium hydroxide. Journal of endodontics, 24(1), 15-17. https://doi.org/10.1016/S0099-2399(98)80205-7.

International Organization for Standardization. Biological Evaluation of Medical Devices: Part 5-Tests for In Vitro Cytotoxicity. Geneva, Switzerland: ISO;2009:10993-5.

Kapralos, V., Koutroulis, A., Ørstavik, D., Sunde, P. T., \& Rukke, H. V. (2018). Antibacterial activity of endodontic sealers against planktonic bacteria and bacteria in biofilms. Journal of Endodontics, 44(1), 149-154. https://doi.org/10.1016/j.joen.2017.08.023.

Lovato, K. F., \& Sedgley, C. M. (2011). Antibacterial activity of endosequence root repair material and proroot MTA against clinical isolates of Enterococcus faecalis. Journal of Endodontics, 37(11), 1542-1546. https://doi.org/10.1016/j.joen.2011.06.022.

Marín-Bauza, G. A., Silva-Sousa, Y. T., da Cunha, S. A., Rached-Junior, F. J., Bonetti-Filho, I., Sousa-Neto, M. D., \& Miranda, C. E. (2012). Physicochemical properties of endodontic sealers of different bases. Journal of applied oral science: revista FOB, 20(4), 455-461. https://doi.org/10.1590/s1678-77572012000400011.

Mendes, A. T., Silva, P., Só, B. B., Hashizume, L. N., Vivan, R. R., Rosa, R., Duarte, M., \& Só, M. (2018). Evaluation of phy sicochemical properties of new calcium silicate-based sealer. Brazilian Dental Journal, 29(6), 536-540. https://doi.org/10.1590/0103-6440201802088.

Parirokh, M., \& Torabinejad, M. (2010). Mineral trioxide aggregate: a comprehensive literature review--Part III: Clinical applications, drawbacks, and mechanism of action. Journal of Endodontics, 36(3), 400-413. https://doi.org/10.1016/j.joen.2009.09.009.

Poggio, C., Dagna, A., Ceci, M., Meravini, M. V., Colombo, M., \& Pietrocola, G. (2017). Solubility and pH of bioceramic root canal sealers: A comparative study. Journal of Clinical and Experimental Dentistry, 9(10), e1189-e1194. https://doi.org/10.4317/jced.54040.

Shakya, V. K., Gupta, P., Tikku, A. P., Pathak, A. K., Chandra, A., Yadav, R. K., Bharti, R., \& Singh, R. K. (2016). An in vitro evaluation of antimicrobial efficacy and flow characteristics for AH Plus, MTA Fillapex, CRCS and Gutta Flow 2 root canal sealer. Journal of Clinical and Diagnostic Research: JCDR, 10(8), ZC104-ZC108. https://doi.org/10.7860/JCDR/2016/20885.8351.

Siboni, F., Taddei, P., Zamparini, F., Prati, C., \& Gandolfi, M. G. (2017). Properties of BioRoot RCS, a tricalcium silicate endodontic sealer modified with povidone and polycarboxylate. International Endodontic Journal, 50 Suppl 2, e120-e136. https://doi.org/10.1111/iej.12856.

Swimberghe, R., Coenye, T., De Moor, R., \& Meire, M. A. (2019). Biofilm model systems for root canal disinfection: a literature review. International Endodontic Journal, 52(5), 604-628. https://doi.org/10.1111/iej.13050.

Tanomaru-Filho, M., Cristine Prado, M., Torres, F., Viapiana, R., Pivoto-João, M., \& Guerreiro-Tanomaru, J. M. (2019). Physicochemical properties and bioactive potential of a new epoxy resin-based root canal sealer. Brazilian Dental Journal, 30(6), 563-568. https://doi.org/10.1590/0103-6440201802861.

Viana, F. L. P., Sampieri, M. B. S., Viana, P. P., Cruz, S. M. L., Vivan, R. R., Duarte, M. A. H., Zanin, I. C. J., \& Vasconcelos, B. C. (2021) Análise do pH e da atividade antimicrobiana de um novo medicamento intracanal biocerâmico Bio-C Temp. Research, Society and Development, 10(7), e33310716550, 2021. DOI: http://dx.doi.org/10.33448/rsd-v10i7.16550.

Zancan, R. F., Canali, L., Tartari, T., Andrade, F. B., Vivan, R. R., \& Duarte, M. (2018). Do different strains of E. faecalis have the same behavior towards intracanal medications in in vitro research? Brazilian Oral Research, 32, e46. https://doi.org/10.1590/1807-3107bor-2018.vol32.0046.

Zordan-Bronzel, C. L., Esteves Torres, F. F., Tanomaru-Filho, M., Chávez-Andrade, G. M., Bosso-Martelo, R., \& Guerreiro-Tanomaru, J. M. (2019). Evaluation of physicochemical properties of a new calcium silicate-based sealer, Bio-C Sealer. Journal of Endodontics, 45(10), 1248-1252. https://doi.org/10.1016/j.joen.2019.07.006. 